\title{
Dietary supplementation with n-3 fatty acids (n-3 FA) for 4 weeks reduces post-exercise fatigue and delayed onset muscle soreness (DOMS) in trained male athletes
}

\author{
L. Benson and S. Mushtaq \\ Department of Clinical Sciences and Nutrition, University of Chester, UK
}

This abstract was presented as the Whole Body Metabolism Theme highlight.

High intensity exercise in the form of eccentric contractions can lead to the formation of free radicals, stimulating an inflammatory response $^{(1,2)}$. Consumption of n-3 FA may help modify inflammation and immune reactions beneficial to health by decreasing interleukin-6, tumour necrosis factor-alpha and C-reactive protein ${ }^{(3)}$. For trained athletes to improve athletic performance, recovery from training is important and DOMS is frequently experienced following eccentric exercise, impacting negatively on strength ${ }^{(4)}$. The Western diet is however, characterised by a high n-6 FA consumption relative to n-3 FA, formulating ratios often in excess of 16:1 ${ }^{(5)}$. The use of non-steroidal anti-inflammatory drugs in the form of n-3 FA has been investigated by a number of clinical trials in untrained athletes, but whether this can be translated into attenuating exercise induced inflammation in trained athletes is still under investigation.

A double-blind, randomised controlled trial was conducted in 22 trained male athletes who supplemented their diet with either $3000 \mathrm{mg} / \mathrm{d}$ of fish oil (gel capsules) consisting of $990 \mathrm{mg}$ eicosapentaenoic acid (EPA) and $660 \mathrm{mg}$ docosahexaenoic acid (DHA) $(n=11)$, or $3000 \mathrm{mg} / \mathrm{d}$ olive oil placebo $(n=11)$, for $28 \mathrm{~d}$. Participants underwent 3 sets of eccentric bicep curls in their dominant arm until failure and arm circumference, number of repetitions completed and DOMS/fatigue scores via visual analogue scale (VAS) were recorded at 0,24 and $48 \mathrm{~h}$ after exercise, pre and post supplementation.

No group performed better during the eccentric bicep test, pre and post supplementation, and at baseline, no differences were observed between groups for DOMS and fatigue. However, post supplementation, DOMS was significantly lower at $24 \mathrm{~h}$ $(\mathrm{P}=0.005)$ and $48 \mathrm{~h}(\mathrm{P}=0.002)$ and fatigue was significantly lower at $24 \mathrm{~h}(\mathrm{P}=0.043)$ and $48 \mathrm{~h}$ post exercise $(\mathrm{P}<0.001)$ in the n-3 FA group compared to the placebo group (Fig. 1). These findings indicate that n-3 FA supplementation has the potential to promote recovery and subsequently increase athletic performance in trained male athletes and may be a useful ergogenic aid. Possible anti-inflammatory mechanisms of n-3 FA should be further investigated using specific biomarkers of inflammation.
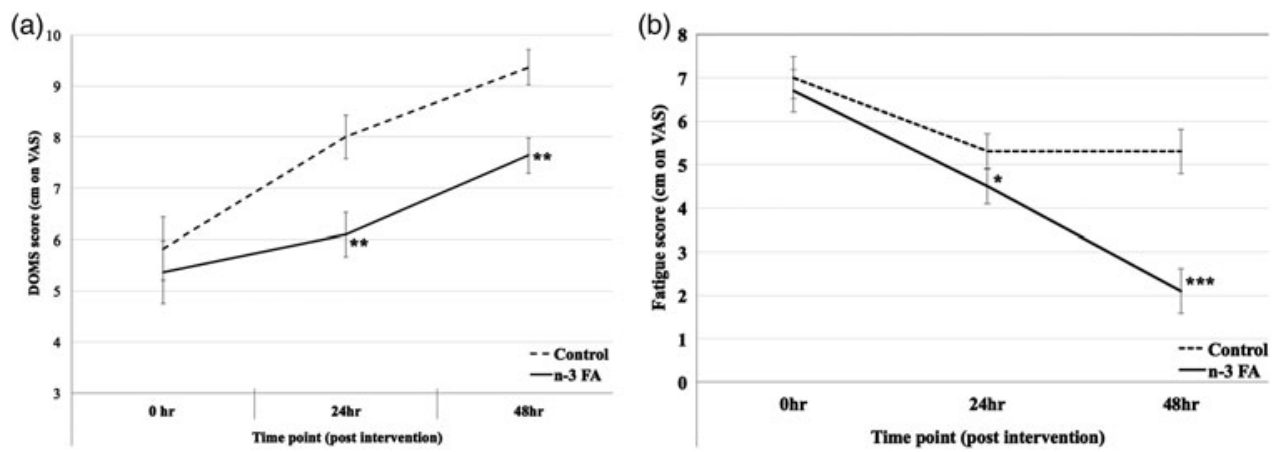

Figure 1. DOMS (a) and fatigue (b) scores (cm on VAS scale) in response to eccentric bicep curls in trained male athletes following 4 weeks supplementation with $3000 \mathrm{mg} / \mathrm{d} \mathrm{n}-3 \mathrm{FA}(\mathrm{n}=11)$ and placebo $(\mathrm{n}=11)$. Asterisks indicate a significant difference in scores between groups at equivalent time points $(* \mathrm{P}<0.05, * * \mathrm{P}<0 \cdot 01, * * * \mathrm{P}<0.001)$.

1. McBride JM, Kraemer WJ Triplett-McBride T, \& Sebastianelli W (1998) Medicine and Science in Sports and Exercise. 30(1) 67-72.

2. Simopoulos AP (2007) Current Sports Medicine Reports 6, 230-236.

3. Simopoulos AP (2002) Biomedicine and Pharmacotherapy 56, 365-79.

4. Ebbeling CB \& Clarkson PM (1989) Sports Medicine 7, 207-34.

5. Pesola GR \& Lawal F (2013) Internet Journal of Asthma, Allergy and Immunology 9, 1. 